\title{
The effects of providing visual feedback and auditory stimulation using a robotic device on balance and gait abilities in persons with stroke: a pilot study
}

\author{
Jae Ho Park', Yijung Chung ${ }^{b}$ \\ aDepartment of Physical Therapy, The Graduate School, Sahmyook University, Seoul, Republic of Korea \\ ${ }^{b}$ Department of Physical Therapy, College of Health and Welfare, Sahmyook University, Seoul, Republic of Korea
}

Objective: The purpose of this study was to investigate the effects of providing visual feedback and auditory stimulation using a robotic device on balance and gait abilities in stroke patients.

Design: Randomized controlled pilot trial.

Methods: Fifteen subjects were randomly divided into three groups where five subjects were in the visual feedback robotic device assist gait training (VRGT) group, five subjects in the auditory stimulation robotic device assist gait training (ARGT) group, and five subjects in the control group. Subjects received visual feedback and auditory stimulation while undergoing robotic gait training for 45 minutes, three times a week for 2 weeks, and all subjects had undergone general physical therapy for 30 minutes, five times a week for 2 weeks. All subjects were assessed with the Berg balance scale (BBS), timed up and go (TUG) test, and 10-meter walking test (10MWT) pre- and post-intervention.

Results: All subjects showed that BBS, TUG test, and 10MWT scores significantly improved post-intervention $(p<0.05)$, and the control group also had significantly improved post-treatment $(p<0.05)$. The VRGT and the ARGT showed significant improvements in BBS, TUG, and 10MWT scores compared with the control group $(p<0.05)$. The VRGT group showed a significant improvement in BBS, TUG, and 10MWT scores compared with the control group $(p<0.05)$. In addition, it has been confirmed that VRGT had significantly improved in BBS, TUG test, and 10MWT scores compared with the auditory stimulation and control group $(p<0.05)$.

Conclusions: The results of this study showed improved balance and gait abilities after VRGT and ARGT groups compared with general physical therapy and was found to be effective in enhancing the functional activity of persons affected with stroke.

Key Words: Gait, Robotics, Stroke, Visual feedback

\section{Introduction}

Stroke represents a variety of neurological symptoms, such as loss of motor function, sensory, cognitive, and language disorders, and symptoms such as muscle weakness, abnormal muscle tone, and reduction of postural control and exercise capacities results in limitations in the performance of functional activities, such as walking [1].

Unlike normal gait, which displays a harmonious move- ment of the limbs and requires minimal energy to produce a smooth and efficient movement of the body, persons affected by stroke have difficulty producing a normal gait and use a variety of compensatory mechanisms. Subsequently, the use of compensatory mechanisms requires more energy consumption and is considered less efficient [2]. In the case of persons with stroke, they display a slower gait cycle, reduced gait speed, differences in step lengths between the affected and unaffected sides, a shortened stance phase of the

Received: 21 August, 2016 Revised: 11 September, 2016 Accepted: 12 September, 2016

Corresponding author: Yijung Chung

Department of Physical Therapy, The Graduate School of Sahmyook University, 815 Hwarang-ro, Nowon-gu, Seoul 01795, Republic of Korea Tel: 82-2-3399-1637 Fax: 82-2-3399-1639 E-mail: yijung36@syu.ac.kr

(c) This is an Open-Access article distributed under the terms of the Creative Commons Attribution Non-Commercial License (http://creativecommons.org/licens es/by-nc/4.0) which permits unrestricted non-commercial use, distribution, and reproduction in any medium, provided the original work is properly cited.

Copyright $@ 2016$ Korean Academy of Physical Therapy Rehabilitation Science 
affected side and relatively prolonged swing phase of the unaffected side, which all pose as a limitation to their functional movement abilities [3]. Thus, recovery of the walking ability of persons affected with stroke can be referred as an essential element in everyday life [4].

In addition, it is necessary for persons with stroke to exercise using appropriate methods for the improvement of walking ability.

Recently, due to development of science and technology, the specialization and sophistication of rehabilitation robotics has also increased [5]. Rather than the repetitive task of performing high-intensity training, robotic rehabilitation allows gait training that is safe [6]. Furthermore, rehabilitation robots allow repetitive tasks of high-intensity strength training for persons with stroke, which assists the reorganization process of the brain tissue and thus activating the neuroplasticity process of the cerebral cortex and the central nervous system, thus improving motor learning of the upper and lower limbs and producing a more efficient gait speed and balance [7]. One type of robot performs on the axis of movement of the distal extremities (end-effector type) and other types have an exo-skeletal structure similar to human skeletal structure enabling the performance of greater rhythmic movements (exo-skeletal type) [8].

Recently, the more widely and actively used is the exoskeletal type robot (Lokomat Pro; Hocoma AG, Volketswil, Switzerland). It consists of a robotic device that controls the treadmill, bodyweight support system, and the movement of the hip and knee [9]. When comparing the robotic device to the general body weight support (BWS) gait training, the training methods of the robotic device are objective and accurate [10]. Currently, research on robot gait rehabilitation training is on the rise, and studies have been actively in progress in various view-points [11].

Robotic rehabilitation training promotes the elicitation of voluntary participation from the patient, which allows them to become more conative [12].

For this reason, robotic gait training with visual feedback is used. The use of visual feedback is critical for the maintenance of balance, and visual ability plays a large role in providing postural stability and identification and regulation of the movement space [13].

In addition, visual feedback is essential for gait activities or avoiding obstacles and regulating the position of the foot in relation to the floor, taking into consideration the limb trajectory and step target location [14].

Virtual reality has been researched as an assessment and treatment tool [2], and provide real-life effects through interaction and feedback, which is capable of acquiring various techniques [15].

When looking at the application of rehabilitation robotics being intended for persons with stroke, Yang et al. [16] found that post-intervention with rehabilitative robotic gait training, changes in foot pressure could be seen, which indicated improved balance and symmetrical gait and Bonnyaud et al. [17] had reported positive effects, such as flexion of affected knee joint, improved gait speed, stride length, and hip extensor strength, after the use of rehabilitative robotic gait training. In addition, a study by Brütsch et al. [18] reported that the use of rehabilitative robotic gait training with use of a virtual reality program together promoted a higher rate of participation, regarding rehabilitative robots as an effective method of training.

However, the current research practices of gait training with robotics combined with virtual reality are insufficient. Therefore, the purpose of this study was to investigate the effects of providing visual feedback and auditory stimulation with robotic gait training on balance and gait abilities in stroke patients, and also to assess the effectiveness of robotic gait-training for rehabilitating patients with hemiplegia.

\section{Methods}

\section{Subjects}

This study included a pretest-posttest control group design where the subjects were divided according into intervention methods, such as the visual feedback robotic device assist gait training (VRGT) group, auditory stimulation robotic device assist gait training (ARGT) group, or control group. Fifteen subjects who were admitted in Chungnam National University Hospital in Daejeon and were receiving exercise therapy had voluntarily agreed to participate after being informed of the study purpose and methods.

The inclusion criteria were as follows: 1) Diagnosis if a stroke greater than 6 months ago, 2) no difficulties with vision or spatiotemporal abilities, 3) mini-mental state examination Korean version (MMSE-K) score of 22 or greater, 4) no diabetic, neurological, or orthopedic issues (Figure 1).

\section{Intervention}

Fifteen subjects who have never experienced robotic gait training participated in the study, and to prevent the effects of spontaneous recovery, subjects with an onset of stroke 6 months ago were included, as well as no multiple diseases, 


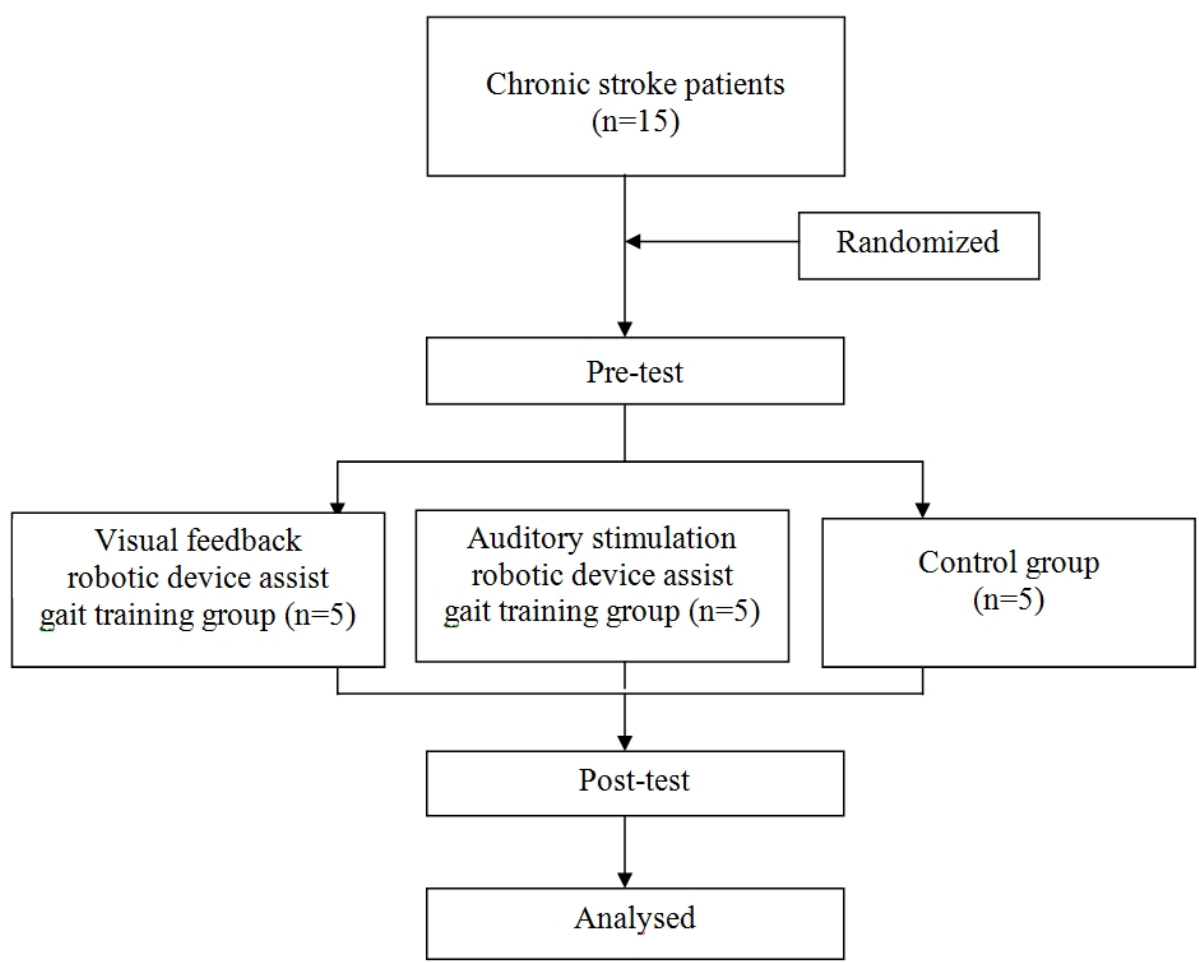

Figure 1. The experimental procedure of this study.

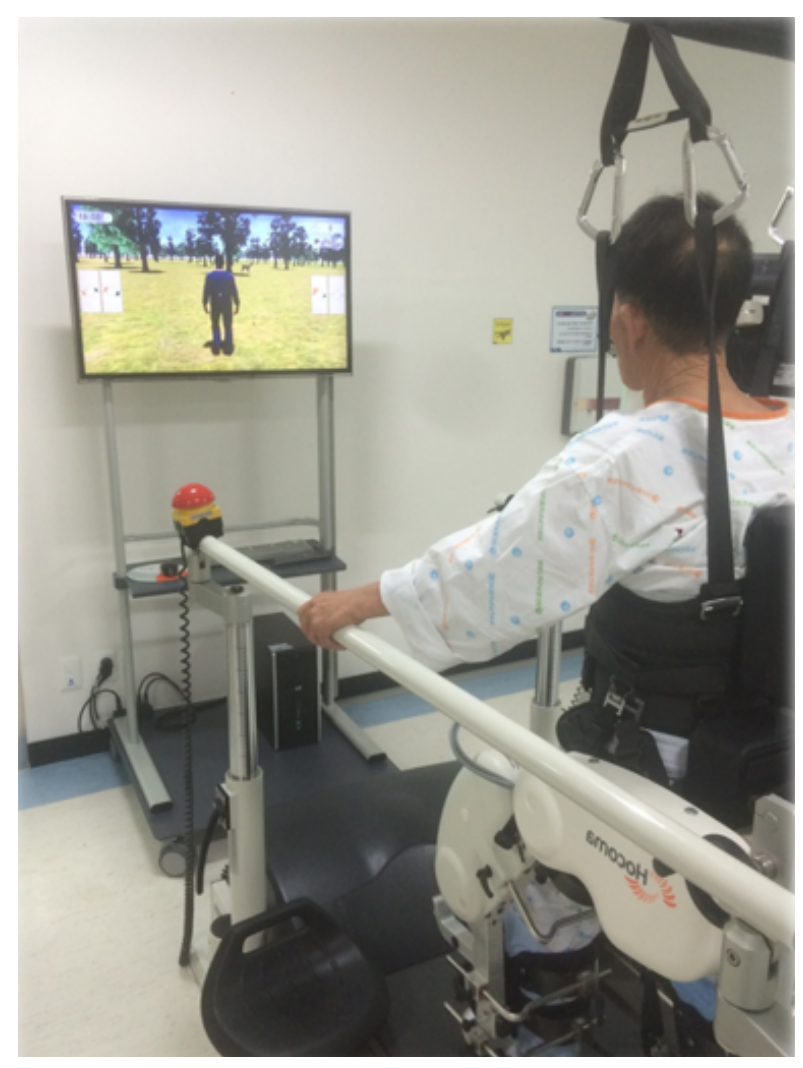

Figure 2. Visual feedback robotic device assisted gait training. and have the ability to comprehend the study contents and follow instructions. In order to decrease the effects of selection bias, subjects were randomly allocated into either experimental group or the control group. The robotic gait-training group received general physical therapy for $30 \mathrm{mi}-$ nutes a day, five times a week for two weeks, with $45 \mathrm{mi}-$ nutes of robotic gait training, three times a week for two weeks. The control group received general physical therapy for 30 minutes, five times a week for two weeks in addition to ground gait training for 30 minutes a day, three times a week for two weeks.

All subjects in each group were assessed for balance and gait abilities prior to the intervention [14]. Using rehabilitation robot was exo-skeletal type robot in this study. The exoskeletal type robot, is similar to a human bone structure (Lokomat Pro), was used due to its ability to effectively promote normal gait patterns [8]. The speed used during the robotic gait training was $1.5-2.0 \mathrm{~km} / \mathrm{h}$ and the BWS was $40 \%-50 \%$ depending on the functional condition of the patients, and a $100 \%$ guidance force during gait training was applied (Figure 2).

The use of augmented feedback virtual reality program of the Lokomat Pro with the VRGT group combined with robotic assisted-gait training is a program that can be applied for various training methods. 
In addition, subjects in the ARGT group had their tempo of gait adjusted using a metronome that can be tailored to the individual subject's step.

To increase the reliability of the trainer and the evaluator, training and assessment was performed by a physical therapist with more than 5 years of experience.

\section{Outcome measures}

\section{Berg balance scale}

The Berg balance scale (BBS) is composed of 14 different items that can quantitatively evaluate the degree of balance and fall risk through direction observation. Items can be classified into three regions of sitting, standing, and postural changes, and each of the 14 items can be scored between 0-4 points, with 56 points being the maximum score. A score of 45 or less indicates the need for the use of a cane or other gait assistive devices, a score of 41-44 indicates a low fall-risk, 21-40 indicates a higher fall risk, and 0-20 indicates a very high risk for falls and injuries. The evaluation assessed dynamic and static balance ability and takes approximately 15 minutes to complete. A therapist with more than 3 years of clinical experience had performed the measurements prior to and after the intervention.

\section{Timed up and go test}

The timed up and go (TUG) test measures the time it takes for a subject to rise from a seated position at the "start" signal, walk up to the $3 \mathrm{~m}$ mark, and then return back to the chair until they are completely seated. It has a reliability of $\mathrm{r}=0.99$ and a high inter-rater reliability of $\mathrm{r}=0.98$. The mean value was obtained from a total of three measurements.

\section{0-meter walking test}

With the presence of gait disabilities due to neurological damage, the 10-meter walking test (10MWT) is a standard test used to investigate the extent of gait ability.

\section{Data analysis}

This study used the PASW Statistics ver. 18.0 program (IBM Co., Armonk, NY, USA). A normality analysis was performed on the general charcteristics of subjects and a paired t-test was performed to examine for changes pre and post-intervention for each group. A one-way ANOVA was used to determine for statistically significant differences in balance and walking ability between groups after two weeks, a post-hoc analysis was performed with the Duncan method, and the significance level was set at $p<0.05$.

\section{Results}

\section{General characteristics and medical characteristics of subjects}

The general characteristics and medical characteristics of all subjects in the VRGT, ARGT, and control groups were all homogenous (Table 1).

Table 1. The general characteristics of participants

$(\mathrm{N}=15)$

\begin{tabular}{|c|c|c|c|c|c|}
\hline Characteristic & VRGT group $(\mathrm{n}=5)$ & ARGT group $(n=5)$ & Control group $(\mathrm{n}=5)$ & $\mathrm{F}$ & $p$ \\
\hline Age (y) & $52.4(12.05)$ & $55.0(5.00)$ & $57.2(11.56)$ & 0.757 & NS \\
\hline Height (cm) & $167.8(8.52)$ & $170.4(6.98)$ & $166.0(5.52)$ & 0.628 & NS \\
\hline Weight (kg) & $65.9(4.85)$ & $67.1(5.05)$ & $68.4(6.65)$ & 0.782 & NS \\
\hline Delay (mo) & $9.2(1.30)$ & $9.2(2.28)$ & $9.0(1.58)$ & 0.979 & NS \\
\hline MMSE-K & $28.2(1.09)$ & $28.6(0.89)$ & $28.4(0.54)$ & 0.775 & NS \\
\hline Gender & & & & 0.804 & NS \\
\hline Male & 3 & 3 & 2 & & \\
\hline Female & 2 & 2 & 3 & & \\
\hline Hemiplegic side & & & & 0.250 & NS \\
\hline Left & 4 & 3 & 3 & & \\
\hline Right & 1 & 2 & 2 & & \\
\hline Mechanism & & & & 0.493 & NS \\
\hline Haemorrhage & 2 & 3 & 4 & & \\
\hline Ischaemia & 3 & 2 & 1 & & \\
\hline
\end{tabular}

Values are presented as mean (SD) or number only.

VRGT: visual feedback robotic device assist gait training, ARGT: auditory stimulation robotic device assist gait training, MMSE-K: minimental state examination Korean version, NS: non-significant. 
Table 2. Changes in balance ability and gait of the participants in this study

$(\mathrm{N}=15)$

\begin{tabular}{|c|c|c|c|c|c|c|c|c|}
\hline \multirow{2}{*}{ Variable } & \multicolumn{2}{|c|}{ VRGT group $(\mathrm{n}=5)$} & \multicolumn{2}{|c|}{ ARGT group $(n=5)$} & \multicolumn{2}{|c|}{ Control group $(\mathrm{n}=5)$} & \multirow{2}{*}{$\mathrm{F}$} & \multirow{2}{*}{$p$} \\
\hline & Pre-test & Post-test & Post-test & Post-test & Pre-test & Post-test & & \\
\hline BBS (score) & $37.6(2.33)$ & $45.2(2.40)$ & $37.0(1.41)$ & $41.2(3.34)$ & $37.4(1.14)$ & $40.0(1.41)$ & 6.997 & 0.001 \\
\hline Difference (post-pre) & \multicolumn{2}{|c|}{$7.6(1.95)^{\mathrm{a}, \mathrm{b}}$} & \multicolumn{2}{|c|}{$4.2(2.68)^{\mathrm{a}}$} & \multicolumn{2}{|c|}{$2.6(0.54)$} & & \\
\hline $\mathrm{t}$ & \multicolumn{2}{|c|}{-7.757} & \multicolumn{2}{|c|}{-3.500} & \multicolumn{2}{|c|}{-10.614} & & \\
\hline$p$ & \multicolumn{2}{|c|}{0.001} & \multicolumn{2}{|c|}{0.025} & \multicolumn{2}{|c|}{0.000} & & \\
\hline TUG test (sec) & $66.4(9.58)$ & $31.6(6.40)$ & $66.2(3.89)$ & $55.4(2.40)$ & $66.6 \pm 7.60$ & $60.8 \pm 9.23$ & 5.845 & 0.006 \\
\hline Difference (post-pre) & \multicolumn{2}{|c|}{$-34.8(11.98)^{\mathrm{a}, \mathrm{b}}$} & \multicolumn{2}{|c|}{$-10.8(3.27)^{\mathrm{a}}$} & \multicolumn{2}{|c|}{$-5.8(3.03)$} & & \\
\hline $\mathrm{t}$ & \multicolumn{2}{|c|}{5.805} & \multicolumn{2}{|c|}{7.383} & \multicolumn{2}{|c|}{4.276} & & \\
\hline$p$ & \multicolumn{2}{|c|}{0.004} & \multicolumn{2}{|c|}{0.002} & \multicolumn{2}{|c|}{0.013} & & \\
\hline 10MWT (sec) & $69.2(2.71)$ & $26.0(7.89)$ & $69.4(2.19)$ & $48.6(3.84)$ & $69.6(3.04)$ & $60.0(4.18)$ & 7.222 & 0.000 \\
\hline Difference (post-pre) & \multicolumn{2}{|c|}{$-43.2(8.63)^{\mathrm{a}, \mathrm{b}}$} & \multicolumn{2}{|c|}{$-20.8(3.19)^{\mathrm{a}}$} & \multicolumn{2}{|c|}{$-9.6(1.51)$} & & \\
\hline $\mathrm{t}$ & \multicolumn{2}{|c|}{10.006} & \multicolumn{2}{|c|}{14.563} & \multicolumn{2}{|c|}{$\begin{array}{r}14.154 \\
0.000\end{array}$} & & \\
\hline$p$ & \multicolumn{2}{|c|}{0.001} & \multicolumn{2}{|c|}{0.000} & \multicolumn{2}{|c|}{0.000} & & \\
\hline
\end{tabular}

Values are presented as mean (SD).

VRGT: visual feedback robotic device assist gait training, ARGT: auditory stimulation robotic device assist gait training, BBS: Berg balance scale, TUG: timed up and go, 10MWT: 10-meter walking test.

${ }^{a}$ Significant difference compared with the control group $(p<0.05)$. ${ }^{\mathrm{b}}$ Significant difference compared with ARGT $(p<0.05)$.

\section{Changes in balance ability and gait of the participants in this study}

The BBS, TUG test, and 10MWT significantly increased in the VRGT and ARGT $(p<0.05)$, and control groups were significantly increased post intervention $(p<0.05)$. All of the outcome measures showed a greater significant increase in VRGT group compared to ARGT group $(p<0.05)$. There was a significant difference in the results when comparing ARGT group with the control group $(p<0.05)$ (Table 2).

\section{Discussion}

This study investigated the effects of visual feedback and auditory stimulation robotic gait-training on balance and gait abilities of 15 persons with stroke. There was a statistically significant improvement in balance and gait abilities of the VRGT and ARGT groups $(p<0.05)$, and also a significant improvement compared with the control group $(p<0.05)$.

With balance being defined as the ability to maintain posture and move without falling [19], and in chronic stroke patients, the presence of gait impairments depend on the presence of balance impairments.

Persons with stroke display a decrease in single leg stance and decrease hip and knee joint angles during the affected side step and the single support time in the stance phase, decreased gait speeds, inappropriate weight-shifting onto the affected side, and also asymmetrical gait [2]. Therefore, in order to display an optimal gait, balance ability is an essential element and plays a big impact on daily life [4].

A study by Dias et al. [20] mentioned that there was a positive impact on the use of robotic devices for rehabilitation on persons affected by stroke, and Wong et al. [21] reported improved in dynamic balance abilities, as demonstrated by the BBS and TUG test scores. Therefore, the BBS and TUG tests were conducted in order to investigate for changes in balance abilities, similar to previous studies. The results of this study showed that all outcome measures had a significant difference pre and post intervention within the VRGT and ARGT groups. Positive effects were noticeable when comparing the experimental with the control groups $(p<$ $0.05)$. It is considered that these changes in gait abilities are influenced by the positive changes in balance abilities.

Bonnyaud et al. [17] reported that after undering rehabilitative robotic gait-training, asymmetrical gait patterns, or in other words, symmetrical hip and knee joint movements has been shown. Sale et al. [22] conducted a study with Parkinson's disease patients and reported that positive changes could be observed in step and stride lengths post intervention with rehabilitative robotic gait-training. Therefore, this study used visual feedback and auditory stimulation to compare its effects on balance and gait abilities. The visual feedback that is provided in virtual reality provides motivation for the patients to produce active movements, improves postural stability [23], and is considered to positively impact the balance and gait abilities in per- 
sons with stroke [24]. Mirelman et al. [3] reported that the use of virtual reality in the background with use of rehabilitative robotic gait-training improved gait speed and other positive effects. A study by Brütsch et al. [18] found that the use of visual stimulation with virtual reality on subjects with gait disabilities induced active participation and a positive impact on movement.

Regular auditory stimulation provides patients with intrinsic feedback in relation to the patient's movement objectives, and thus, auditory stimulation produces improved muscle activation patterns and internal control abilities, phases of regular auditory feedback leads to more control and activation of motor neurons in a consistent and synchronized manner [25].

Therefore, in order to promote active participation and movement of subjects, robotic gait-training with auditory stimulation has been applied. The results have indicated that improved balance and gait abilities post usage of robotic gait training with auditory stimulation has a positive impact $(p<$ $0.05)$. Based on the results, it is considered that use of virtual reality with visual and auditory stimulation and with robotic gait-training provides active movement of the affected lower extremity, improve muscular strength, and simultaneously create improved balance, leading to a more symmetrical gait pattern.

In addition, repetitive task-oriented training with application of the robotic gait-training assists to accelerate the remapping process of the cerebral cortex immediately, creating long-term changes [25]. Also, performing repetitive task-oriented training facilitates neuroplasticity through neurological reorganization and, vitalizing the cerebral cortex and central nervous system, composed of exercising the limbs, thus having a positive impact on gait speed and gait abilities [26].

Clinically, improvement of gait abilities is the primary interest of rehabilitation research in persons affected by hemiplegic stroke [4], and gait speed can be used as a barometer for the ability to perform independent walking and performance of daily life activities [1].

A recent study by Yang et al. [16] has highly reported that persons affected with stroke who had participated in robotic gait-training had displayed a more balanced and stable gait pattern. Drużbicki et al. [27] conducted a study involving rehabilitative robotic gait-training for children with cerebral palsy and had found a significant effect on gait speed. Mirelman et al. [3] reported that after the application of rehabilitative robotic training, there was an improvement in gait speeds due to increase ankle joint muscle strength.

When comparing the outcome measures between the experimental group with the robotic gait-training and the control group, there was a positive impact on the capacity to perform the 10MWT and TUG test $(p<0.05)$.

It can also be considered that the overall exercise function of the lower extremity has improved, and that there is consistently a positive impact of rehabilitative gait-training on not only balance and gait abilities, but also on improving exercise function. Compiling all the results together, it can be stated that the use of visual and auditory stimulation combined with robotic gait-training on persons affected with stroke does influence balance and gait abilities. Through this study, improved balance and gait abilities of persons with chronic stroke has been observed after participating in a rehabilitative robotic gait-training program.

\section{Conflict of Interest}

The authors declared no potential conflicts of interest with respect to the authorship and/or publication of this article.

\section{References}

1. Poli P, Morone G, Rosati G, Masiero S. Robotic technologies and rehabilitation: new tools for stroke patients' therapy. Biomed Res Int 2013;2013:153872.

2. Perry J. Gait analysis: normal and pathological function. Thorofare (NJ): Slack; 1992.

3. Mirelman A, Patritti BL, Bonato P, Deutsch JE. Effects of virtual reality training on gait biomechanics of individuals post-stroke. Gait Posture 2010;31:433-7.

4. Krishnan C, Kotsapouikis D, Dhaher YY, Rymer WZ. Reducing robotic guidance during robot-assisted gait training improves gait function: a case report on a stroke survivor. Arch Phys Med Rehabil 2013;94:1202-6.

5. Esquenazi A, Packel A. Robotic-assisted gait training and restoration. Am J Phys Med Rehabil 2012;91(11 Suppl 3):S217-27; quiz S228-31.

6. Kwakkel G, Kollen B, Lindeman E. Understanding the pattern of functional recovery after stroke: facts and theories. Restor Neurol Neurosci 2004;22:281-99.

7. Mehrholz J, Werner C, Kugler J, Pohl M. Electromechanical-assisted training for walking after stroke. Cochrane Database Syst Rev 2007;(4):CD006185.

8. Mehrholz J, Pohl M. Electromechanical-assisted gait training after stroke: a systematic review comparing end-effector and exoskeleton devices. J Rehabil Med 2012;44:193-9.

9. Hidler J, Neckel N. Inverse-dynamics based assessment of gait using a robotic orthosis. Conf Proc IEEE Eng Med Biol Soc 2006; $1: 185-8$. 
10. Riener R, Lünenburger L, Jezernik S, Anderschitz M, Colombo G, Dietz V. Patient-cooperative strategies for robot-aided treadmill training: first experimental results. IEEE Trans Neural Syst Rehabil Eng 2005;13:380-94.

11. Tefertiller C, Pharo B, Evans N, Winchester P. Efficacy of rehabilitation robotics for walking training in neurological disorders: a review. J Rehabil Res Dev 2011;48:387-416.

12. Schück A, Labruyère R, Vallery H, Riener R, Duschau-Wicke A. Feasibility and effects of patient-cooperative robot-aided gait training applied in a 4-week pilot trial. J Neuroeng Rehabil 2012; 9:31.

13. Shumway-Cook A, Anson D, Haller S. Postural sway biofeedback: its effect on reestablishing stance stability in hemiplegic patients. Arch Phys Med Rehabil 1988;69:395-400.

14. Reynolds RF, Day BL. Visual guidance of the human foot during a step. J Physiol 2005;569:677-84.

15. Kang YJ, Ku J, Han K, Kim SI, Yu TW, Lee JH, et al. Development and clinical trial of virtual reality-based cognitive assessment in people with stroke: preliminary study. Cyberpsychol Behav 2008;11:329-39.

16. Yang JK, Ahn NE, Kim DH, Kim DY. Plantar pressure distribution during robotic-assisted gait in post-stroke hemiplegic patients. Ann Rehabil Med 2014;38:145-52.

17. Bonnyaud C, Pradon D, Boudarham J, Robertson J, Vuillerme N, Roche N. Effects of gait training using a robotic constraint (Lokomat $($ ) on gait kinematics and kinetics in chronic stroke patients. J Rehabil Med 2014;46:132-8.

18. Brütsch K, Koenig A, Zimmerli L, Mérillat-Koeneke S, Riener $\mathrm{R}$, Jäncke L, et al. Virtual reality for enhancement of robot-assisted gait training in children with central gait disorders. J Rehabil Med 2011;43:493-9.

19. Shumway-Cook A, Horak FB. Assessing the influence of sen- sory interaction of balance. Suggestion from the field. Phys Ther 1986;66:1548-50.

20. Dias D, Laíns J, Pereira A, Nunes R, Caldas J, Amaral C, et al. Can we improve gait skills in chronic hemiplegics? A randomised control trial with gait trainer. Eura Medicophys 2007;43: 499-504.

21. Wong CK, Bishop L, Stein J. A wearable robotic knee orthosis for gait training: a case-series of hemiparetic stroke survivors. Prosthet Orthot Int 2012;36:113-20.

22. Sale P, De Pandis MF, Le Pera D, Sova I, Cimolin V, Ancillao A, et al. Robot-assisted walking training for individuals with Parkinson's disease: a pilot randomized controlled trial. BMC Neurol 2013;13:50.

23. Jang SH, You SH, Hallett M, Cho YW, Park CM, Cho SH, et al. Cortical reorganization and associated functional motor recovery after virtual reality in patients with chronic stroke: an experimenter-blind preliminary study. Arch Phys Med Rehabil 2005;86:2218-23.

24. Thaut MH, McIntosh GC, Rice RR. Rhythmic facilitation of gait training in hemiparetic stroke rehabilitation. J Neurol Sci 1997; 151:207-12.

25. Jang SH, Kim YH, Cho SH, Lee JH, Park JW, Kwon YH. Cortical reorganization induced by task-oriented training in chronic hemiplegic stroke patients. Neuroreport 2003;14:137-41.

26. Harvey RL. Improving poststroke recovery: neuroplasticity and task-oriented training. Curr Treat Options Cardiovasc Med 2009; 11:251-9.

27. Drużbicki M, Rusek W, Snela S, Dudek J, Szczepanik M, Zak E, et al. Functional effects of robotic-assisted locomotor treadmill thearapy in children with cerebral palsy. J Rehabil Med 2013;45: 358-63. 